\title{
Modification and mechanical properties of electrospun blended fibermat of PLGA and siloxane-containing vaterite/PLLA hybrids for bone repair
}

\author{
G. Poologasundarampillai, K. Fujikura, A. Obata, T. Kasuga* \\ Department of Frontier Materials, Nagoya Institute of Technology, Gokiso-cho, Showa-ku, Nagoya 466-8555, Japan
}

Received 17 February 2011; accepted in revised form 11 April 2011

\begin{abstract}
Multi-syringe electrospinning has been successfully employed to produce a blended fibermat composed of poly(lactic-glycolic acid) (PLGA) fibers and a composite fiber for bone repair. The composite fiber, siloxane-containing vaterite $(\mathrm{SiV}) /$ poly(L-lactic acid) (PLLA), donated as SiPVH has the ability to release soluble silica species and calcium ions at a controlled rate. The SiPVH fibermats have demonstrated excellent bone regeneration ability in vivo at the front midline of the calvaria of rabbits. However, they are brittle and have low tensile strength resulting from the large particulate $\mathrm{SiV}(60 \mathrm{wt} \%)$ content. In this study, co-electrospinning of PLGA with SiPVH was performed in the hope of achieving a blended fibermat with improved mechanical properties. The co-electrospun fibermats showed good homogeneous blending of the PLGA and SiPVH composite fibers that had excellent flexibility. The blended PLGA-SiPVH fibermats had significantly improved mechanical properties compared to the SiPVH fibermats, where more than 20 times higher elongation to failure was achieved on comparison to the SiPVH fibermat. As well as strength, high porosity and large pore size are vital for the migration of cells into the centre of the graft. This was accomplished by heating the PLGA-SiPVH fibermats at $110^{\circ} \mathrm{C}$ for a fixed time, which induced the softening and flow of PLGA towards the more stable SiPVH fibers. Heating had successfully produced PLGA-SIPVH fibermats with large open pores and inter-fused SiPVH fibers, which also had better tensile mechanical properties than the SiPVH fibermat.
\end{abstract}

Keywords: biopolymers, biocomposites, electrospinning, blended fibermat, mechanical properties

\section{Introduction}

Large maxillofacial defects are often regenerated with barrier membranes [1-3]. Barrier membranes function by containing the regeneration site from ingression of soft tissue into the defect site, which is often filled with bone harvested from the patients and/or biological agents such as bone forming cells and growth factors [3]. These, also called guided bone regeneration (GBR) membranes are required to be biocompatible, integrate with host-surrounding tissue and have high mechanical properties and flexibility to maintain and support defect site and should also be easy to handle.

\footnotetext{
${ }^{*}$ Corresponding author, e-mail: kasuga.toshihiro@nitech.ac.jp (c) BME-PT
}

Fibrous membranes are promising as GBR materials due to their high and breathable interconnected porosity [3-8]. This allows efficient transport of nutrient and oxygen into and metabolic waste out of the regeneration site. The fibrous structure enhances cell attachment [9], therefore promotes soft tissue growth along the membrane while the porosity can be designed to inhibit in-growth. Electrospinning is a simple and versatile method to produce fibermats with fiber diameters ranging from nano-micrometer [10-12]. Electrospun materials are very promising for the regeneration of tissues and organs [13-15] due to the possibility of mimicking some of the 
structural features of natural extra cellular matrix (ECM) [16]. Numerous studies show the enhanced cellular attachment and proliferation on the fibrous materials $[9,17,18]$. Several studies have also shown the higher mechanical properties, especially the elongation to failure, of the electrospun fibermats [19-22] and blended fibermats [23]. The mechanical properties of electrospun fibers depends greatly on the degree of alignment of the fibers within the mat, fiber lay-ups and interface properties of fiberfiber contact $[20,24]$. Therefore, the versatility of electrospinning presents a promising method of fiber production for biomedical application.

The current authors have previously employed electrospinning to fabricate bi-layered GBR membranes that were shown to be bioactive, biocompatible and mechanically strong while being flexible [25]. The bi-layered membranes consists of two layers, a PLLA fibermat layer and a siloxane-containing vaterite $(\mathrm{SiV}) /$ PLLA composite fibermat layer (SiPVH) $[25,26]$. The PLLA layer acted as the soft tissue barrier membrane, where the interconnected porosity was optimised by tailoring the fiber diameter to inhibit cell migration into the depth of the fibermat. The SiPVH fibermat layer provides space for bone formation. Both, the PLLA and the SiPVH layers were bonded together by hot-pressing with a stainless steel mesh that was placed on top of the PLLA layer. Whereby, at regions on the fibermat in contact with the steel mesh fiber-fiber fusion was induced [26]. Early experiments on SiPVH dipcoated membranes showed MC3T3-E1 cells proliferate on the membrane and more so on the hydroxyl carbonate apatite (HCA) coated SiPVH [5]. Therefore, the authors had also coated the bi-layered membranes with a bone mineral-like apatite layer by soaking in simulate body fluid (SBF). The authors then performed in vivo experiments on New Zealand rabbits by placing the bi-layered GBR membranes at the $8 \mathrm{~mm}$ defects in the front midline of the calvaria of the rabbits [25]. They found enhanced bone formation on the SiVPVH composite layer at the sites where depressions (regions of fiber-fiber fusion) were made my stainless steel mesh. Their study of the fibermat morphology showed a unique melted structure which they proposed could have influenced the cells to proliferate and lay-down new bone faster at those sites $[25,26]$. They also noted that the cells could only migrate through the non- depressed areas; therefore porosity for cell migration was important [25].

From an enhancement of bone regeneration point of view; it is highly desirable to obtain the melt-fused morphology throughout the fibermat, however the hot-pressing process closes majority of the pores, which are critical for cell migration. The hot-pressing process that leads to the desired unique morphology is also the major drawback in this material. Therefore innovative processing methods are required to overcome this hurdle in achieving the unique morphology. Here, in this study one such strategy is explored. The fabrication of the unique melted structure throughout the fibermat was attempted by electrospinning a blended fibermat and applying a heating procedure that induced partial melting and fusion of the poly(lactic glycolic acid) (PLGA) fibers. In addition to the melt-fused morphology, by co-spinning PLGA and SiPVH, the blended fibermat was expected to have improved mechanical properties, which is important for ease of handling, shaping to specific shape and finally to support the biological moieties and cells during the process of bone regeneration.

\section{Experimental section}

PLLA (Purasorb ${ }^{\circledR}$; PURAC, The Netherlands) and PLGA (53\% lactic, 47\% glycolic) (Purasorb $^{\circledR}$; PURAC, The Netherlands) with average molecular weight $\left(M_{\mathrm{w}}\right)$ of $260 \mathrm{kDa}$ and $160 \mathrm{kDa}$, respectively were used. Chloroform was purchased from Wako Pure Chemical Industries, Ltd., Osaka, Japan.

\subsection{Preparation of $\mathrm{SiV}$ particles}

The silicon-doped calcium carbonate (SiV) powders were prepared by a carbonation process as described by Yasue et al. [27] and later modified by Kasuga et al. [28]. Briefly, calcium hydroxide $\left(\mathrm{Ca}(\mathrm{OH})_{2}\right)$ and aminopropyltriethoxysilane (APTES) were mixed together in methanol, followed by a constant bubbling of $\mathrm{CO}_{2}$ gas for $75 \mathrm{~min}$ at a rate of $21 \cdot \mathrm{min}^{-1}$. The resulting slurry was dried at $110^{\circ} \mathrm{C}$, resulting in the formation of $\mathrm{SiV}$ powder with average particle size of $1 \mu \mathrm{m}$.

\subsection{Preparation of PLGA-SiPVH blended fibermats}

The SiPVH fibermats were prepared according to the procedure described by Obata et al. [25]. Briefly, 
SiV particles containing fibers, SiPVH, was produced by electrospinning a composite solution of $60 \mathrm{wt} \% \mathrm{SiV}$ and $40 \mathrm{wt} \%$ PLLA. The composite solution was prepared by melt blending $\mathrm{SiV}$ and PLLA at $200^{\circ} \mathrm{C}$ for $10 \mathrm{~min}$ with a kneader and dissolved in chloroform with $10 \mathrm{wt} \%$ polymer concentration. Electrospun fibers of PLGA were produced after dissolving in chloroform with $10 \mathrm{wt} \%$ polymer concentration. Samples were then electrospun on the Nanofiber Electrospinning Unit (NEU, Kato Tech Co, Japan). The mixtures for electrospinning were loaded into a glass syringe and pushed out at a flow rate of $0.50 \mu \mathrm{l} \cdot \mathrm{s}^{-1}$ through a metallic needle (22 gauge for SiPVH and 19 gauge for PLGA) that was connected to a $+20 \mathrm{kV}$ electric field. The fibers were collected on a rotating drum that was positioned $15 \mathrm{~cm}$ from the tip of the needle. Blended fibermats were produced by electrospinning two separate solutions side-by-side. Blended fibermats containing fibers of PLGA-SiPVH were produced with a final thickness of $230 \mu \mathrm{m}$ after spinning for $210 \mathrm{~min}$.

\subsection{Modification of blended fibermats}

PLGA-SiPVH fibermats were cut into circular membranes with a diameter of $17 \mathrm{~mm}$. These were heated in a drying oven at 90,110 and $130^{\circ} \mathrm{C}$ for 15 , 20, 25 and $30 \mathrm{~min}$ to obtain the heat-fused morphology of the PLGA-SiPVH fibermats. Previous work has demonstrated the enhanced cellular proliferation of MC3T3 cells on an HA coated SiPVH fibermat in comparison to an uncoated SiPVH fibermats [26]. Therefore, cut fibermats before and after heating were soaked in a solution containing ions with 1.5 times the concentration of conventional simulated body fluid (SBF) to obtain the HA coating. The 1.5 SBF solution consisting of $3.75 \mathrm{mM} \mathrm{Ca}^{2+}$, $213.0 \mathrm{mM} \mathrm{Na}^{+}, 2.25 \mathrm{mM} \mathrm{Mg}^{2+}, 7.5 \mathrm{mM} \mathrm{K}^{+}$, $222.45 \mathrm{mM} \mathrm{Cl}^{-}, 6.3 \mathrm{mM} \mathrm{HCO}_{3}^{-}, 1.5 \mathrm{mM} \mathrm{HPO}_{4}{ }^{2-}$, $0.75 \mathrm{mM} \mathrm{SO}_{4}{ }^{2-}, 50 \mathrm{mM}\left(\mathrm{CH}_{2} \mathrm{OH}\right)_{3} \mathrm{CNH}_{2}$ and $45.0 \mathrm{mM} \mathrm{HCl}$ was prepared using reagent grade $\mathrm{NaCl}, \mathrm{NaHCO}_{3}, \mathrm{KCl}, \mathrm{K}_{2} \mathrm{PO}_{4} \cdot 3 \mathrm{H}_{2} \mathrm{O}, \mathrm{MgCl}_{2} \cdot 6 \mathrm{H}_{2} \mathrm{O}$, $\mathrm{HCl}, \mathrm{CaCl}_{2}, \mathrm{NaSO}_{4}$ and $\mathrm{NH}_{2} \mathrm{C}\left(\mathrm{CH}_{2} \mathrm{OH}\right)_{3}$. After immersion in $1.5 \mathrm{SBF}$ solution for $24 \mathrm{~h}$ the membranes were washed with distilled water and dried at room temperature.

\subsection{Characterisation of blended fibermats}

Samples for scanning electron microscopic (SEM, JSM-6301F, JEOL, Japan) observations were coated with amorphous osmium using an Os coater (Neoc, Meiwafosis, Japan) and observed under $5 \mathrm{kV}$ accelerating voltage and $15 \mathrm{~mm}$ working distance.

Tensile tests were performed on the AGS-G, Shimadzu, Japan machine equipped with a $50 \mathrm{~N}$ load cell. Rectangular samples with dimensions of $5 \mathrm{~mm} \times$ $40 \mathrm{~mm}$ were cut out of the fibermats and tested with a grip-to-grip separation of $20 \mathrm{~mm}$ at a strain rate of $5 \mathrm{~mm} / \mathrm{min}$. Samples were tested until complete failure and SEM observations were performed on the failed samples.

\section{Results and discussion}

Figure 1 shows the SEM images of the PLGASiPVH blended fibermats. The SiPVH on comparison to PLGA fibers has a much rougher fibrous surface due to the presence of $\mathrm{SiV}$ particles in the SiPVH fibers. The SiPVH fibers also have a large average fiber diameter than the PLGA, this was due to the higher viscosity of the SiPVH $(5312 \mathrm{mPa} \cdot \mathrm{s})$ electrospinning solution than PLGA (793 $\mathrm{mPa} \cdot \mathrm{s})$. The fiber diameters measured from the SEM images of PLGA ranged from 1.5-10.0 $\mu \mathrm{m}$ and the average diameter of SiPVH was measured to be $18 \pm 5 \mu \mathrm{m}$. The fiber diameter of SiPVH was found to be more homogeneous than that of PLGA. The cross-sectional image of the PLGA-SiPVH fibermat (Figure $1 b$ ) shows that the polymer and SiPVH fibers were very well blended.

\subsection{Heating of PLGA-SiPVH blended fibermats}

Optical microscopy was performed on the samples heated at 90,110 and $130^{\circ} \mathrm{C}$ for 15 and $30 \mathrm{~min}$ (data not shown). The PLGA-SiPVH fibermat heated at $90^{\circ} \mathrm{C}$ for $30 \mathrm{~min}$ showed only a very small change in original morphology. On the other hand, at $130^{\circ} \mathrm{C}$ complete melting and large run off of the PLGA fibers were observed at both 15 and 30 min of heating. Figure 2 shows the SEM images of the PLGASiPVH fibermats after heating at $110^{\circ} \mathrm{C}$ for 15,20 , 25 and $30 \mathrm{~min}$. All the PLGA-SiPVH fibermats heated at $110^{\circ} \mathrm{C}$ showed PLGA softening and flow; 


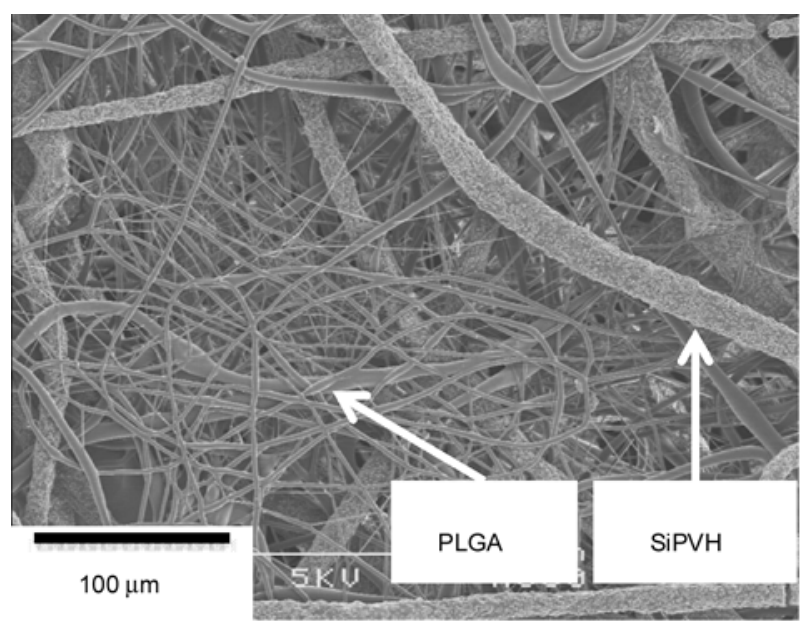

a)

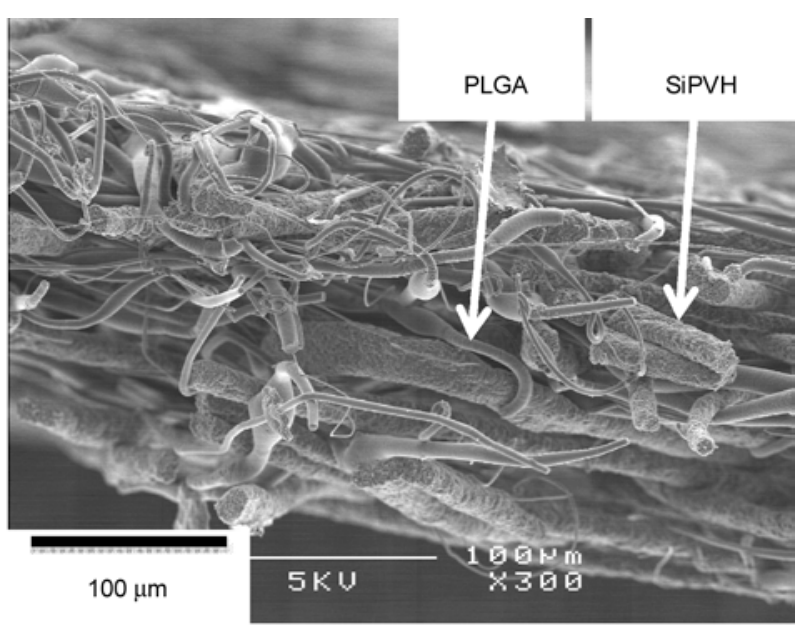

b)

Figure 1. Surface (a) and cross-sectional (b) SEM images of the PLGA-SiPVH fibermat

this lead to fusion with the SiPVH fibers. An identical morphology was observed after heating for 15 and $20 \mathrm{~min}$, here the initial as-spun PLGA fibrous network is still vaguely visible, more so in the 15 min heated sample. However, more coalescence

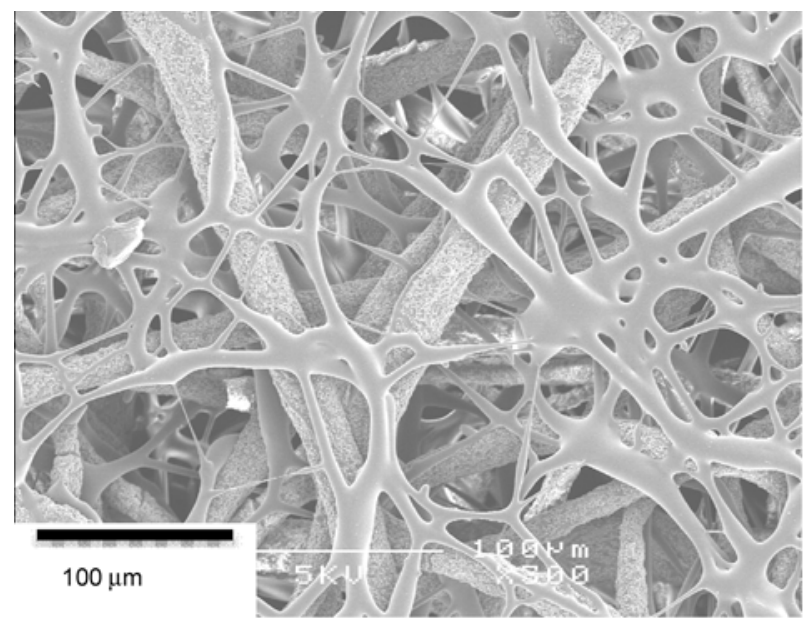

a)

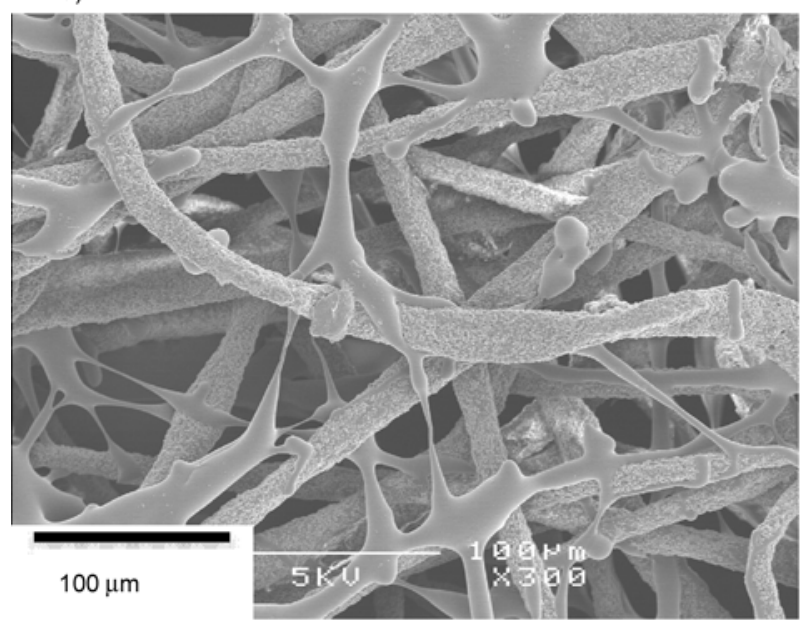

c) of the PLGA was observed on the 20 min heated sample. On the other hand, heating for $30 \mathrm{~min}$ produced a large amount of flow where almost all the PLGA has aggregated towards the SiPVH fibers. However, heating for $25 \mathrm{~min}$ seems to have pro-

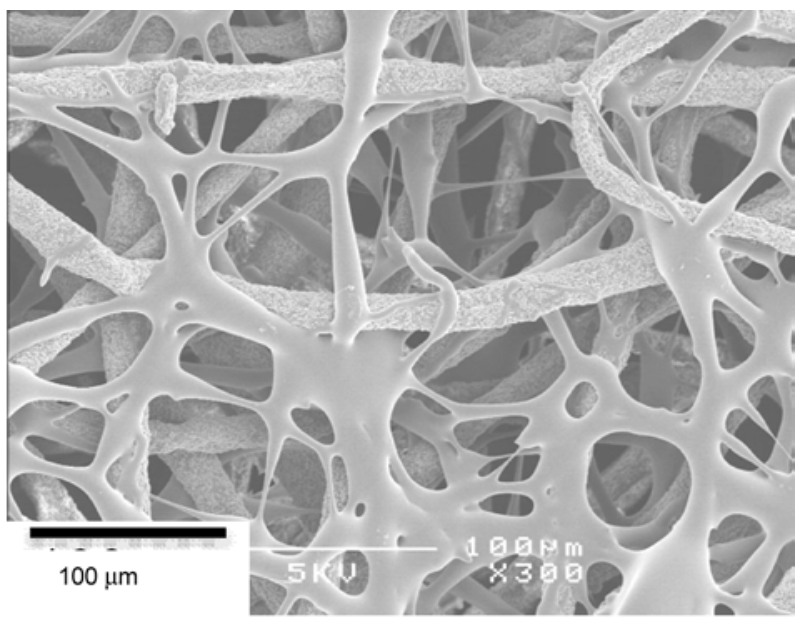

b)

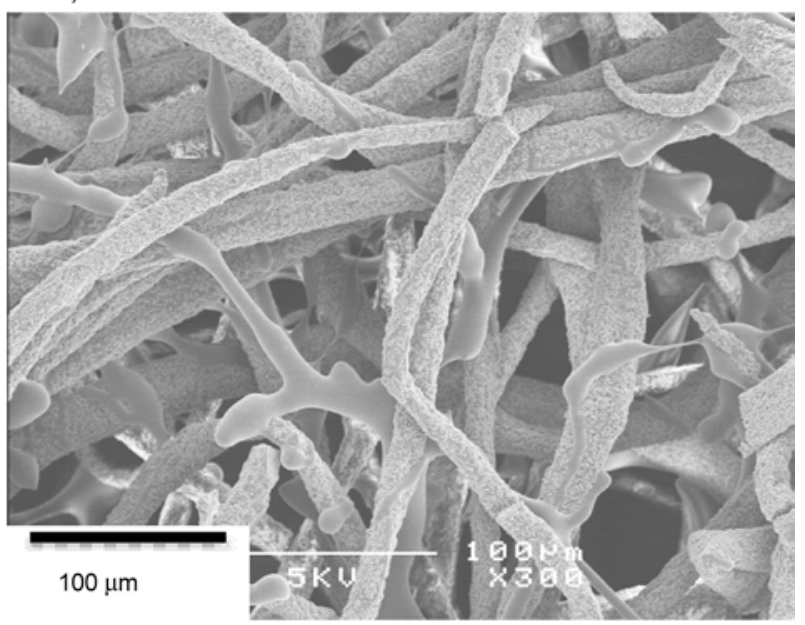

d)

Figure 2. SEM images of the PLGA-SiPVH fibermat after heating at $110^{\circ} \mathrm{C}$ for (a) 15 , (b) 20 , (c) 25 and (d) $30 \mathrm{~min}$ 
duced enough flow of PLGA fibers that has resulted in a fibermat morphology that has open pores of the order of $100 \mu \mathrm{m}$ and a substantial proportion of PLGA still bridging the SiPVH fibers. This morphology could be beneficial for cell attachment, spreading and migration within the fibermat.

\subsection{Morphology of the PLGA-SiPVH blended fibermats soaked in 1.5 SBF}

Figure 3 shows the SEM images of the PLGASiPVH fibermats (Figure 3a) after soaking in 1.5 SBF for $24 \mathrm{~h}$, (Figure 3b, d, e) after heating at $110^{\circ} \mathrm{C}$ for $25 \mathrm{~min}$ then soaking in $1.5 \mathrm{SBF}$ and (Figure $3 \mathrm{c}$ ) after soaking in $1.5 \mathrm{SBF}$ for $24 \mathrm{~h}$ then heating at $110^{\circ} \mathrm{C}$ for $25 \mathrm{~min}$. Firstly, the SiPVH fibers in all of the samples after soaking $1.5 \mathrm{SBF}$ for $24 \mathrm{~h}$ were completely coated with a thick layer of HA (as shown in Figure 3d). As well, several particles of HA were also observed on the surface of PLGA, especially in the case of fine PLGA fibers that were

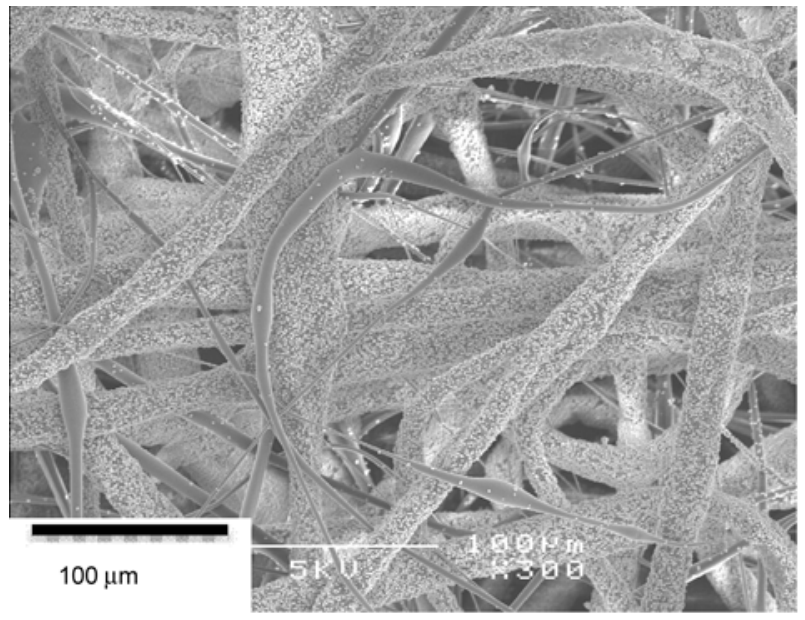

a)

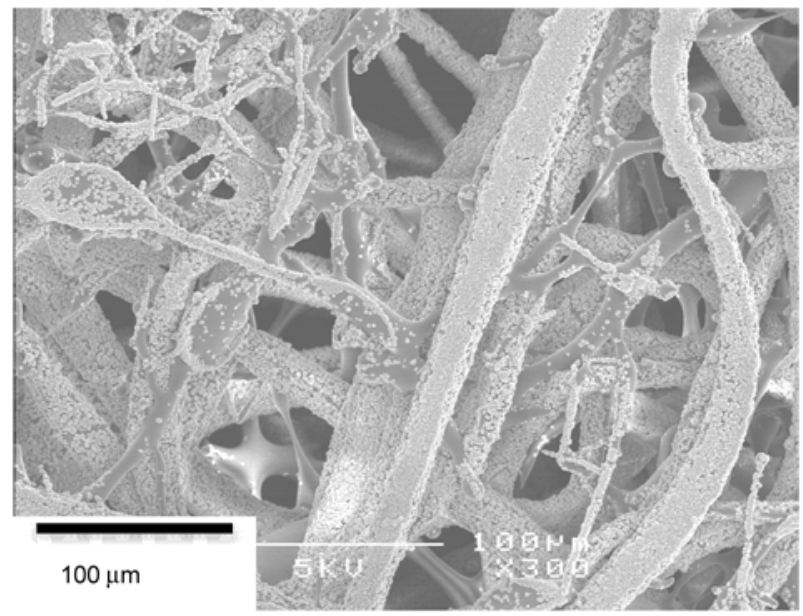

c)

Figure 3. SEM images of the HA-coated PLGA-SiPVH fibermat (a) without heat application, (b) after heating and soaking, (c) after soaking and then heating and (d, e) high magnification images of (b) observed to be completely coated (Figure 3e). Figure 3d, e shows the high magnification SEM images of Figure 3b; the HA particles on the SiPVH and PLGA surface have a globular cauliflower-like morphology. Secondly, the fibrous morphology of the as-spun-HA-coated (Figure $3 \mathrm{a}$ ) and the heated and HA coated (Figure 3b, c) fibermats show a clear difference on the size of the pores in the fibermats. Where, from the SEM images the pore size seems to increase in the order as follows: as-spunHA-coated (Figure 3a) < HA-coated-heated (Figure $3 \mathrm{c})<$ heated-HA-coated (Figure 3b). The throughpore area fraction of the fibermats was calculated from the SEM images in Figure 3 using ImageJ. They were found to be $6.4,16.2$ and $9.2 \%$ for the as-spun-HA-coated, heated-HA-coated and HAcoated-heated, respectively. Although this not the true porosity of the fibermat but it gives an indication of the extent of porosity of each sample and demonstrates that the heating treatment signifi-

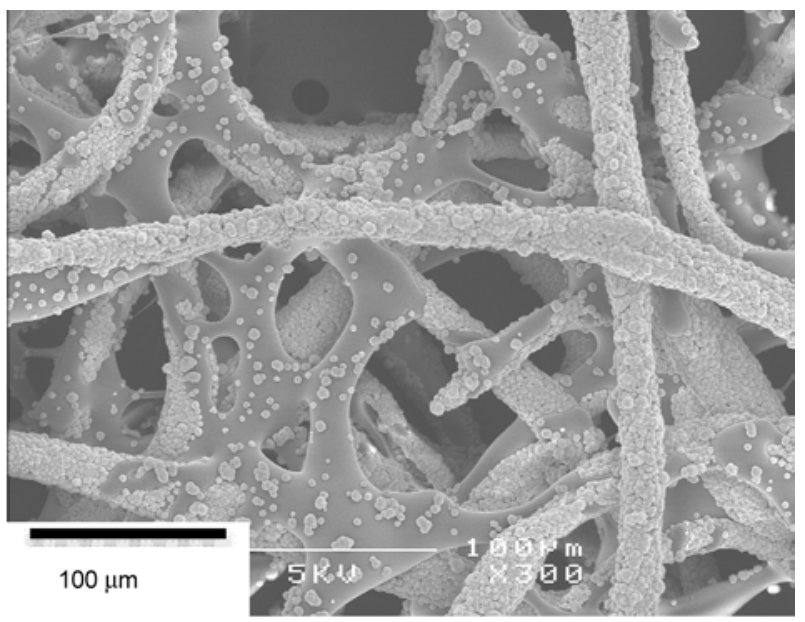

b)
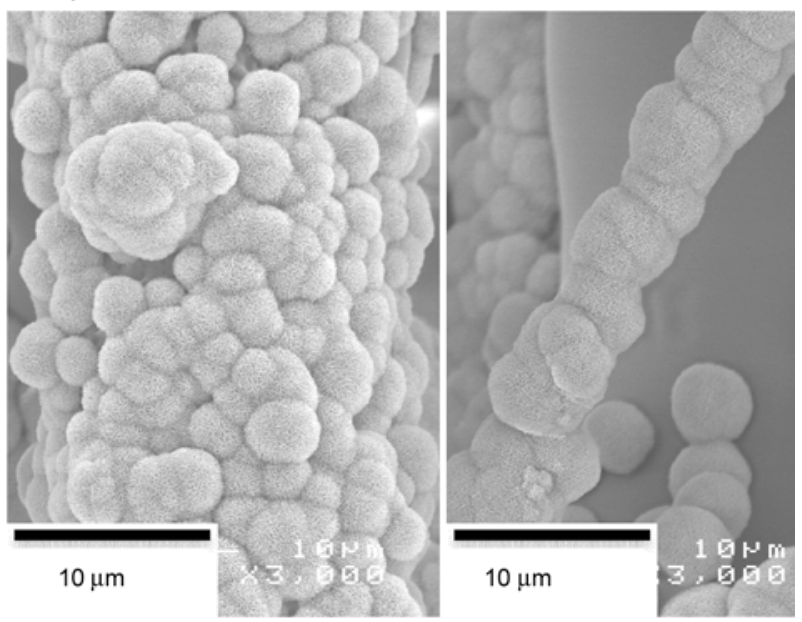

e) 
cantly increases the porosity. The as-spun-HAcoated has the smallest pore space, this is expected since no heating was applied to result in the coalescence of the fine fibers of PLGA. While, in the case of the HA-coated-heated fibermat (Figure 3c) the HA coating on the fine PLGA fibers, where several fine PLGA fibers with HA coating (circled in Figure $3 \mathrm{c}$ ) are clearly visible in the SEM image, has hindered its flow at the post-heating stage. This has reduced the coalescence of PLGA and hence the required effect of heating was not achieved. However, the heated-HA-coated fibermat (Figure 3b) has the desired morphology, where the SiPVH fibers are completely coated with HA and large interfiber distances can be seen. Therefore, for the blended PLGA-SiPVH fibermats, the processing conditions of heating at $110^{\circ} \mathrm{C}$ for $25 \mathrm{~min}$ followed by soaking in $1.5 \mathrm{SBF}$ for $24 \mathrm{~h}$ is proposed to be ideal in achieving the desired fibermat morphology.

\subsection{Mechanical properties of the PLGA-SiPVH blended fibermats}

Figure 4a shows the tensile stress-strain curves and Figure $4 \mathrm{~b}$ ultimate tensile strength (UTS) and elongation to failure of the as-spun SiPVH and blended PLGA-SiPVH fibermats and the heated PLGASiPVH fibermats. Both, the UTS and elongation before failure of all the PLGA-SiPVH blended samples were higher than that of SiPVH fibermats. The SiPVH fibermat had low UTS of $0.16 \mathrm{MPa}$ and a brittle failure with a very low elongation to failure of $1.44 \%$. The as-spun PLGA-SiPVH blended fiber- mat showed the largest elongation before failure of more than $30 \%$. Both, as-spun and the 15 min heated samples had the highest UTS ( 0.5 MPa), which then steadily decreased with heating time. Samples heated for 20 and $25 \mathrm{~min}$ at $110^{\circ} \mathrm{C}$ and then soaked in $1.5 \mathrm{SBF}$ for $24 \mathrm{~h}$ were also tested in tension. A small decrease in the UTS was observed with soaking in SBF. This could be due to the degradation of PLGA in the 1.5 SBF solution, however the drop in UTS was minor.

The elongation to failure of the PLGA-SiPVH blended fibermats decreased drastically after heating, however still higher than that of SiPVH fibermat. Although, the 15 min-heated sample had relatively high UTS, the elongation before failure decreased to around 4\%. Wei et al. [24] showed that the fracture energy decreases with increasing fiberfiber fusion, where in this study longer heating time leads to higher fusion hence the elongation before failure is observed to decrease. Figure 5 shows the SEM images of the failure regions on the as-spun and heated samples of the PLGA-SiPVH blended fibermats after tensile testing. In the as-spun fibermat (Figure 5a-c) large plastic deformation before complete failure is evident, where several regions (circled in Figure 5a) of fiber stretching were observed on the whole fibermat. A higher magnification image of the circled area in Figure 5a is shown in Figure 5b, where the SiPVH fibers were seen to be completely fractured while the PLGA fibers have thinned to various thicknesses. Complete fracture of the sample took place when the cir-
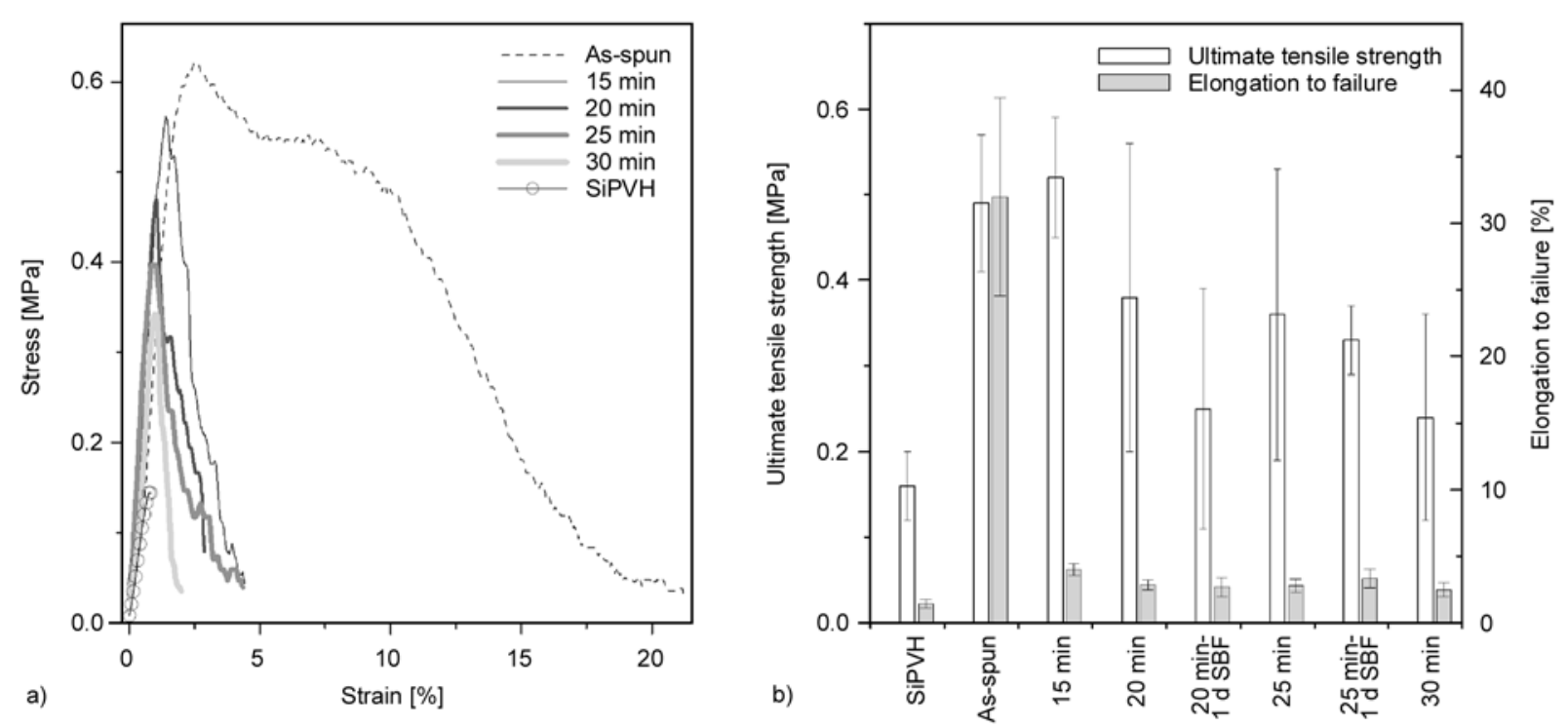

Figure 4. (a) Exemplary tensile stress-strain curves and (b) ultimate tensile strength and elongation to failure of the SiPVH fibermat and PLGA-SiPVH blended fibermats before and after heat treatment at $110^{\circ} \mathrm{C}$ for $15,20,25$ and 30 min. 
cle regions connected up along the width of the whole fibermat. Figure $5 \mathrm{c}$ shows a SEM image from the region of complete failure, which shows more thinned PLGA and fractured SiPVH fibers.

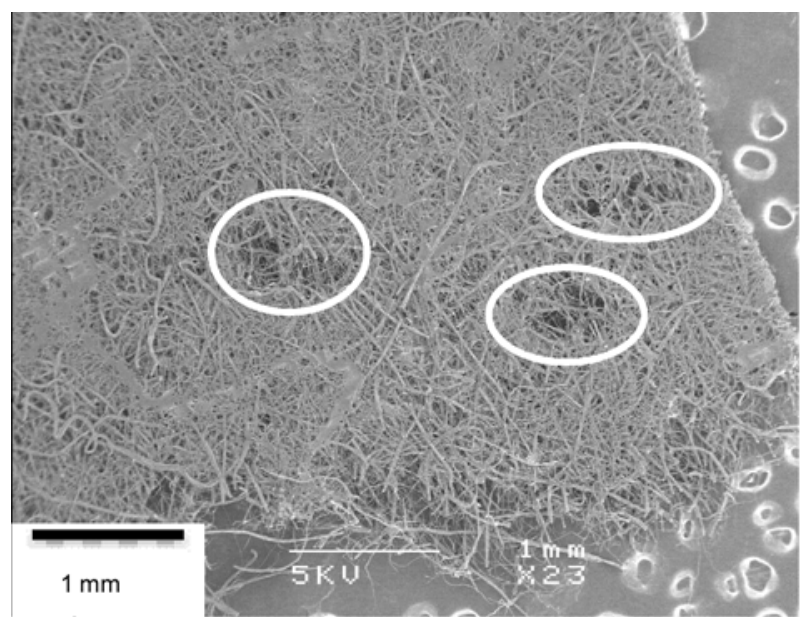

a)

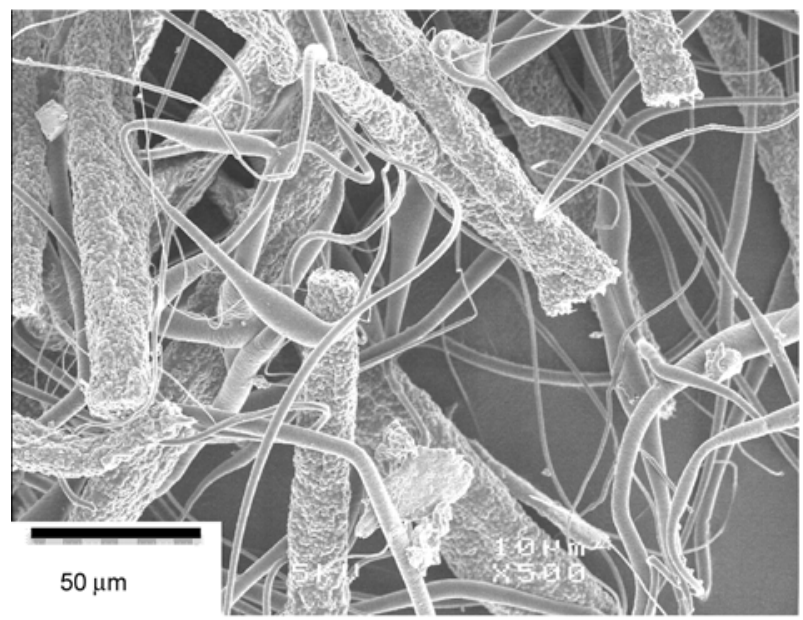

c)

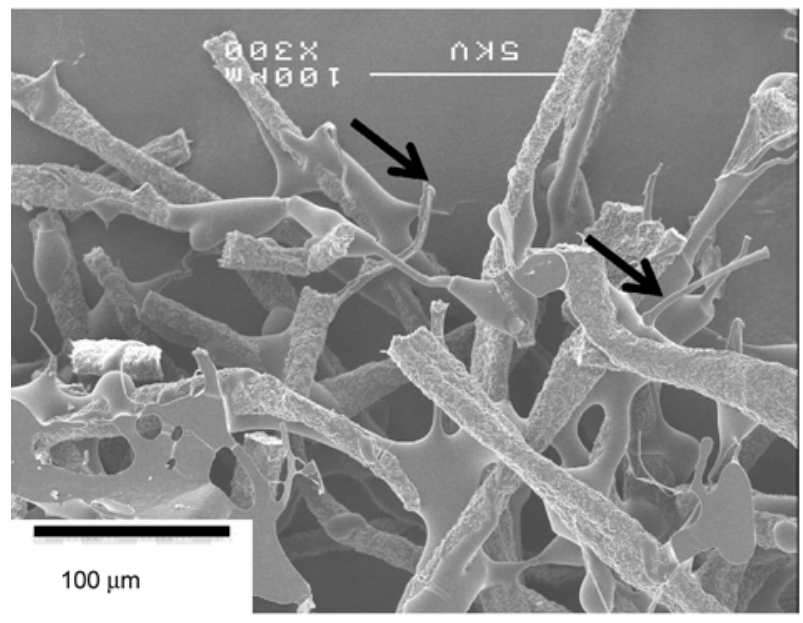

e)
All the heated samples had a similar failure mechanism which was very different from that of the asspun blended fibermats. Plastic deformation in the heated fibermats was observed to be very local and

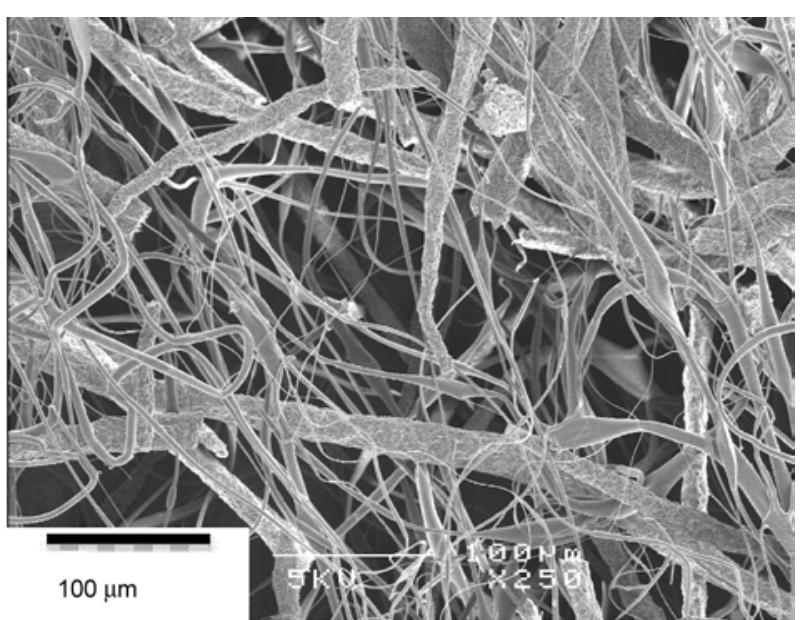

b)

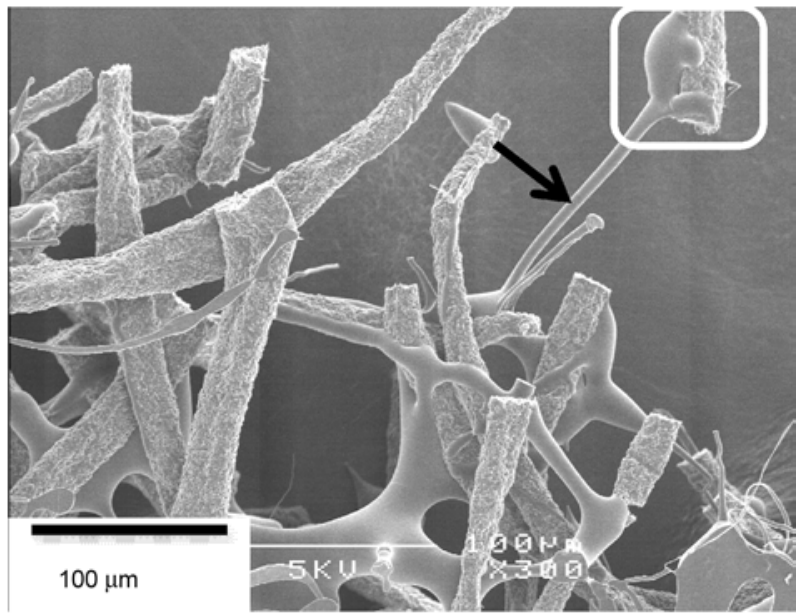

d)

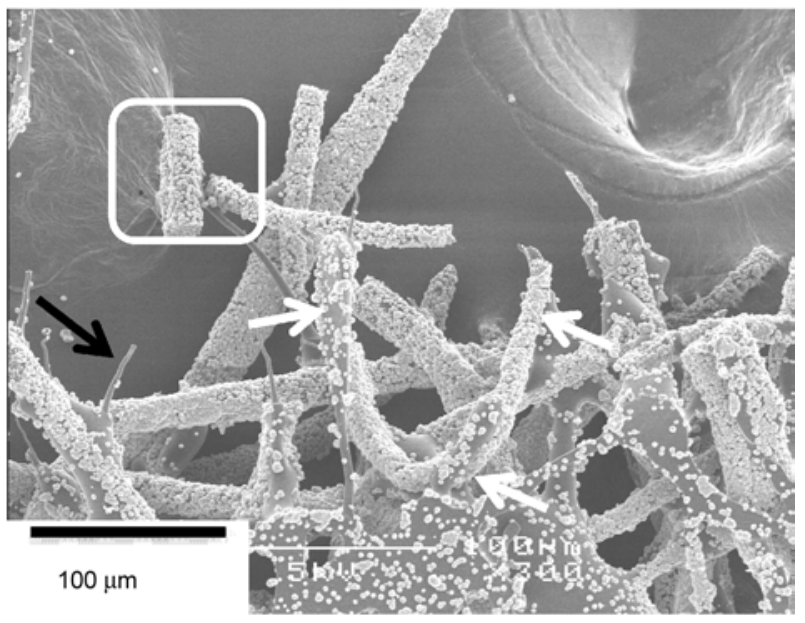

Figure 5. SEM images of the (a-c) as-spun and heated (d) $15 \mathrm{~min}$, (e) $25 \mathrm{~min}$ and (f) 25 min-heated and soaked in $1.5 \mathrm{SBF}$ PLGA-SiPVH fibermats after performing tensile tests. In the images the regions of local deformation in the asspun PLGA-SiPVH are shown with ellipses, box in (d) and (f) show the fractured SiPVH fibers stabilised by PLGA, black arrows point to the PLGA strands that had plastically deformed and white arrows in (f) indicate a segment of SiPVH fiber stabilised by melt-fused PLGA. 
confined to the region of final fracture. Figure $5 \mathrm{~d}-\mathrm{f}$ shows the SEM images of the failure zones of the heated samples. There are several important observations to be made. First, the fiber morphology close to the fracture zone had minimal disturbance after testing, a clear example for this is seen in Figure 5f, where a part of SiPVH fiber shaped ' $U$ ' (indicated by white arrows in SEM image) was not pulled out during testing. This indicates that the failure was brittle-like and that the melt-fused PLGA-SiPVH structure was very stable. Second, small strands of PLGA, indicated by black arrows in the SEM images, are the only PLGA polymer to undergo plastic deformation. Thirdly, these strands of PLGA are observed to hold the fractured pieces of SiPVH fibers in place (square boxes in SEM images), this is advantages after implantation if the SiPVH fibers were to fracture off; they could be held in implantation site by the PLGA. Finally, the failure region and mode of failure of the heated-HA-coated PLGA-SiPVH fibermat (Figure $5 \mathrm{f}$ ) was identical to the hated-only fibermats. Therefore, the melt-fused PLGA-SiPVH fiber morphology obtained by heating at $110^{\circ} \mathrm{C}$ for 20 or $25 \mathrm{~min}$ and HA coated by soaking in $1.5 \mathrm{SBF}$ could be promising for cell adhesion, spreading and migration. In vitro cell culture and in vivo animal studies are required to confirm this.

\section{Conclusions}

An attempt to mimic the morphology of the hot mesh-pressed bi-layered fibermat structure via a double syringe electrospinning and heating process was performed. The SEM images show clearly that the double syringe electrospinning of PLGA and SiPVH results in a well-blended fibrous structure. The PLGA-SiPVH fibermats have more than 30\% elongation before failure and $\sim 0.5 \mathrm{MPa}$ ultimate tensile strength. On heating the PLGA fibers soften and flow towards the SIPVH fibers and adhere to it. This is found to indirectly, increase the pore size of the fibermat and result in multiple triple-points that connect the SiPVH fibers. Heating at $110^{\circ} \mathrm{C}$ for $20 \mathrm{~min}$ and more so after $25 \mathrm{~min}$ was found to produce a microstructure with pores of the order of $100 \mu \mathrm{m}$. The melt-fused PLGA-SiPVH fibermats after heating have higher elongation to failure and ultimate tensile strength on comparison to SiPVHonly fibermats. The melt-fusing by PLGA was also observed to provide stability to the SiPVH fibers while also increasing their strength.

\section{Acknowledgements}

This work was supported in part by Grant-in-Aids (B) (\#20390499) from Japan Society for the Promotion of Science (JSPS), for Scientific Research in Institute of Ceramics Research and Education, NITECH and the JSPS International Training Program.

\section{References}

[1] Mayfield L., Nobréus N., Attström R., Linde A.: Guided bone regeneration in dental implant treatment using a bioabsorbable membrane. Clinical Oral Implants Research, 8, 10-17 (1997). DOI: $10.1111 / \mathrm{j} .1600-0501.1997 . t b 00002 . x$

[2] Eickholz P., Kim T-S., Holle R.: Regenerative periodontal surgery with non-resorbable and biodegradable barriers: Results after 24 months. Journal of Clinical Periodontology, 25, 666-676 (1998). DOI: 10.1111/j.1600-051X.1998.tb02504.X

[3] Yeh H-C., Hsu K-W.: Guided bone regeneration for fenestration defects in dental implants. Chang Gung Medical Journal, 26, 684-689 (2003).

[4] Fujihara K., Kotaki M., Ramakrishna S.: Guided bone regeneration membrane made of polycaprolactone/calcium carbonate composite nano-fibers. Biomaterials, 26, 4139-4147 (2005).

DOI: 10.1016/j.biomaterials.2004.09.014

[5] Maeda H., Kasuga T., Hench L. L.: Preparation of poly(L-lactic acid)-polysiloxane-calcium carbonate hybrid membranes for guided bone regeneration. Biomaterials, 27, 1216-1222 (2006). DOI: 10.1016/j.biomaterials.2005.08.010

[6] Lee E-J., Teng S-H., Jang T-S., Wang P., Yook S-W., Kim H-E., Koh Y-H.: Nanostructured poly( $\varepsilon$-caprolactone)-silica xerogel fibrous membrane for guided bone regeneration. Acta Biomaterialia, 6, 3557-3565 (2010). DOI: $10.1016 /$ j.actbio.2010.03.022

[7] Piattelli A., Scarano A., Paolantonio M.: Bone formation inside the material interstices of e-PTFE membranes: A light microscopical and histochemical study in man. Biomaterials, 17, 1725-1731 (1996). DOI: 10.1016/0142-9612(96)87653-8

[8] Amano Y., Ota M., Sekiguchi K., Shibukawa Y., Yamada S.: Evaluation of a poly-1-lactic acid membrane and membrane fixing pin for guided tissue regeneration on bone defects in dogs. Oral Surgery, Oral Medicine, Oral Pathology, Oral Radiology and Endodontics, 97, 155-163 (2004). DOI: $10.1016 /$ j.tripleo.2003.09.009

[9] Stevens M. M., George J. H.: Exploring and engineering the cell surface interface. Science, 310, 1135-1138 (2005).

DOI: $10.1126 /$ science. 1106587 
[10] Reneker D. H., Chun I.: Nanometre diameter fibres of polymer, produced by electrospinning. Nanotechnology, 7, 216-223 (1996).

DOI: $10.1088 / 0957-4484 / 7 / 3 / 009$

[11] Li D., Xia Y.: Electrospinning of nanofibers: Reinventing the wheel? Advanced Materials, 16, 1151-1170 (2004).

DOI: 10.1002/adma.200400719

[12] Bhardwaj N., Kundu S. C.: Electrospinning: A fascinating fiber fabrication technique. Biotechnology Advances, 28, 325-347 (2010).

DOI: $10.1016 /$ j.biotechadv.2010.01.004

[13] Liang D., Hsiao B. S., Chu B.: Functional electrospun nanofibrous scaffolds for biomedical applications. Advanced Drug Delivery Reviews, 59, 1392-1412 (2007). DOI: $10.1016 /$ j.addr.2007.04.021

[14] Li W-J., Laurencin C. T, Caterson E. J., Tuan R. S., Ko F. K.: Electrospun nanofibrous structure: A novel scaffold for tissue engineering. Journal of Biomedical Materials Research Part A, 60, 613-621 (2002).

DOI: $10.1002 / \mathrm{jbm} .10167$

[15] Zhang Y. Z., Su B., Venugopal J., Ramakrishna S., Lim C. T.: Biomimetic and bioactive nanofibrous scaffolds from electrospun composite nanofibers. International Journal of Nanomedicine, 2, 623-638 (2007).

[16] Brightman A. O., Rajwa B. P., Sturgis J. E., McCallister M. E., Robinson J. P., Voytik-Harbin S. L.: Timelapse confocal reflection microscopy of collagen fibrillogenesis and extracellular matrix assembly in vitro. Biopolymers, 54, 222-234 (2000).

DOI: $10.1002 / 1097-0282(200009) 54: 3<222:: A I D-$ BIP80>3.0.CO;2-K

[17] Xie J., Li X., Xia Y.: Putting electrospun nanofibers to work for biomedical research. Macromolecular Rapid Communications, 29, 1775-1792 (2008).

DOI: $10.1002 /$ marc. 200800381

[18] Jang J-H., Castano O., Kim H-W.: Electrospun materials as potential platforms for bone tissue engineering. Advanced Drug Delivery Reviews, 61, 1065-1083 (2009).

DOI: $10.1016 /$ j.addr.2009.07.008

[19] Wong S-C., Baji A., Leng S.: Effect of fiber diameter on tensile properties of electrospun poly( $\varepsilon$-caprolactone). Polymer, 49, 4713-4722 (2008).

DOI: $10.1016 /$ j.polymer.2008.08.022
[20] Baji A., Mai Y-W., Wong S-C., Abtahi M., Chen P.: Electrospinning of polymer nanofibers: Effects on oriented morphology, structures and tensile properties. Composites Science and Technology, 70, 703-718 (2010).

DOI: 10.1016/j.compscitech.2010.01.010

[21] Lu J-W., Zhang Z-P., Ren X-Z., Chen Y-Z., Yu J., Guo $Z$-X.: High-elongation fiber mats by electrospinning of polyoxymethylene. Macromolecules, 41, 3762-3764 (2008).

DOI: $10.1021 / \mathrm{ma} 702881 \mathrm{k}$

[22] Chandrasekaran A. R., Venugopal J., Sundarrajan S., Ramakrishna S.: Fabrication of a nanofibrous scaffold with improved bioactivity for culture of human dermal fibroblasts for skin regeneration. Biomedical Materials, 6, 015001/1-015001/10 (2011).

DOI: $10.1088 / 1748-6041 / 6 / 1 / 015001$

[23] Kim Y. B., Cho D., Park W. H.: Enhancement of mechanical properties of $\mathrm{TiO}_{2}$ nanofibers by reinforcement with polysulfone fibers. Materials Letters, 64, 189-191 (2010).

DOI: $10.1016 /$ j.matlet.2009.10.038

[24] Wei X., Xia Z., Wong S-C., Baji A.: Modelling of mechanical properties of electrospun nanofibre network. International Journal of Experimental and Computational Biomechanics, 1, 45-57 (2009).

DOI: $10.1504 /$ IJECB.2009.022858

[25] Obata A., Hotta T., Wakita T., Ota Y., Kasuga T.: Electrospun microfiber meshes of silicon-doped vaterite/ poly(lactic acid) hybrid for guided bone regeneration. Acta Biomaterialia, 6, 1248-1257 (2010).

DOI: $10.1016 /$ j.actbio.2009.11.013

[26] Wakita T., Obata A., Poologasundarampillai G., Jones J. R., Kasuga T.: Preparation of electrospun siloxanepoly(lactic acid)-vaterite hybrid fibrous membranes for guided bone regeneration. Composites Science and Technology, 70, 1889-1893 (2010).

DOI: $10.1016 /$ j.compscitech.2010.05.014

[27] Yasue T., Kojima Y., Arai Y.: Preparation of vaterite and control of its crystal shape (in Japanese). Gypsum Lime, 247, 471-480 (1993).

[28] Kasuga T., Maeda H., Kato K., Nogami M., Hata K-I., Ueda M.: Preparation of poly(lactic acid) composites containing calcium carbonate (vaterite). Biomaterials, 24, 3247-3253 (2003). DOI: $10.1016 / \mathrm{S} 0142-9612(03) 00190-\mathrm{X}$ 